OU-HET 521

\title{
Lepton flavor violation in Higgs boson decays under the rare tau decay results
}

\author{
Shinya Kanemura,,$*$ Toshihiko Ota, ${ }^{1,+}$ and Koji Tsumura, ${ }^{1,+}$ \\ ${ }^{1}$ Department of Physics, Osaka University, Toyonaka, Osaka 560-0043, Japan
}

\begin{abstract}
We study lepton flavor violation (LFV) associated with tau leptons in the framework of the two Higgs doublet model, in which LFV couplings are introduced as a deviation from Model II Yukawa interaction. Parameters of the model are constrained from experimental results and also from requirements of theoretical consistencies such as vacuum stability and perturbative unitarity. Current data for rare tau decays provide substantial upper limits on the LFV Yukawa couplings in the large $\tan \beta$ region $(\tan \beta \gtrsim 30)$, which are comparable with predictions in fundamental theories. Here $\tan \beta$ is the ratio of vacuum expectation values of the two Higgs doublets. We show that a search for the LFV decays $\phi^{0} \rightarrow \tau^{ \pm} \mu^{\mp}\left(\tau^{ \pm} e^{\mp}\right)$ of neutral Higgs bosons $\left(\phi^{0}=h, H\right.$ and $\left.A\right)$ at future collider experiments can be useful to further constrain the LFV couplings especially in the relatively small $\tan \beta$ region ( $\tan \beta \lesssim 30$ ), where rare tau decay data cannot give any strong limit.
\end{abstract}

PACS numbers: 11.30.Hv, 12.60.Fr, 14.60.Fg

Keywords: lepton flavor violation, extended Higgs sector, collider physics

*Electronic address: kanemu@het.phys.sci.osaka-u.ac.jp

${ }^{\dagger}$ Electronic address: toshi@het.phys.sci.osaka-u.ac.jp

${ }^{\ddagger}$ Electronic address: ko2@het.phys.sci.osaka-u.ac.jp 


\section{INTRODUCTION}

Experimental determination of the electroweak symmetry breaking sector is important not only to confirm the Higgs mechanism and the mass generation mechanism for matter but also to obtain information for physics beyond the standard model (SM). Lots of new physics models predict extended Higgs sectors with more than one scalar doublets in the low energy effective theories. Such extended Higgs sectors would show distinctive features from the SM phenomenology. The most obvious evidence is the confirmation of the existence of the extra scalar states such as CP-odd and charged states. Even when they are too heavy to be directly detected and only the lightest Higgs boson is found at experiments, we can explore a possibility of the extended Higgs sector by looking for deviations from the SM predictions in its couplings with gauge bosons and fermions as well as in the self coupling. Moreover, it can also be examined by searching for non-SM interactions.

Lepton flavor violation (LFV) is an example for such non-SM phenomena. In particular, LFV in the Yukawa sector can only appear for extended Higgs sectors. Flavor violation between electrons and muons [1] has been tested through rare muon decays such as $\mu \rightarrow e \gamma$ and $\mu \rightarrow e e^{+} e^{-}$, as well as through $\mu-e$ conversion. Tau lepton associated LFV has also been studied by rare decays of tau leptons such as $\tau \rightarrow \ell_{i} P^{0}[2], \tau \rightarrow \ell_{i} M^{+} M^{\prime-}[\underline{3}$, [4], $\tau \rightarrow \ell_{i} \ell^{\prime+} \ell^{\prime-}$ [5, [6], and $\tau \rightarrow \ell_{i} \gamma\left[\right.$ [7, 8], 9], where $\ell_{i}(i=1,2)$ respectively represent an electron and a muon, $P^{0}$ does $\pi^{0}, \eta$ and $\eta^{\prime}$ mesons, $M^{ \pm}\left(M^{\prime \pm}\right)$ does $\pi^{ \pm}$and $K^{ \pm}$mesons, and $\ell^{ \pm}=e^{ \pm}$ and $\mu^{ \pm}$. The LFV Yukawa couplings can be constrained from the data for these processes especially those with the Higgs boson mediation. For $\mu-e$ mixing, the Higgs boson mediated LFV coupling has been discussed in Ref. 10, 11]. Tau lepton associated LFV processes with the Higgs boson mediation have been discussed in models with supersymmetry (SUSY) 12, 13, 14, 15] as well as in the two Higgs doublet model (THDM) in some specific scenarios 16, 17, 18]. In Ref. [19], tau associated LFV processes have been discussed comprehensively in the framework of 4-Fermi contact interactions. Phenomenological consequences of the LFV Yukawa couplings associated with tau leptons have also been studied for future observables such as $B_{s}$ decays 13, 20] at (super) B factories 21] and Higgs boson decays [15, 24, 25] at CERN LHC [26], an electron-positron linear collider (LC) 27] and a muon collider [28]. In addition, it has been pointed out that deep inelastic scattering processes $\mu N \rightarrow \tau X[29$, 30] from intense high energy muons at neutrino factories (or muon colliders) and $e N \rightarrow \tau X[\underline{30}]$ 
by using the electron (positron) beam of a LC would be useful to further explore the tau lepton associated LFV Yukawa couplings.

In this paper, we study LFV in Higgs boson decays into a $\tau-\ell_{i}$ pair in the general framework of the THDM. In order to evaluate possible maximal values of the branching fractions, we first study experimental upper limits on the tau lepton associated LFV Yukawa couplings. The parameter space is tested by theoretical requirements for vacuum stability [31] and perturbative unitarity [32, 33, 34]. Current data from electroweak precision measurements at LEP [35, 36] and those at the B factories [37, 38] also strongly constrain parameters of the Higgs potential. Under these theoretical bounds and experimental limits on the model, possible maximal values of the LFV couplings of $\tau-\ell_{i}-\phi^{0}$ are obtained by using the current data for rare tau decays, where $\phi^{0}$ represents two CP-even $(h$ and $H)$ and a CP-odd $(A)$ Higgs bosons. We then evaluate branching ratios of $\phi^{0} \rightarrow \tau^{ \pm} \ell_{i}^{\mp}$ with the maximal allowed values of the LFV couplings of $\tau-\ell_{i}-\phi^{0}$ in a wide range of the parameter space.

We here consider the model in which Higgs self-interactions and quark Yukawa interactions are invariant under the discrete symmetry $\left(\Phi_{1} \rightarrow+\Phi_{1}\right.$ and $\Phi_{2} \rightarrow-\Phi_{2}$ with $\Phi_{a}$ ( $a=1,2)$ being the Higgs doublets) in order to suppress flavor changing neutral current (FCNC) 39]. If the discrete symmetry is exact, only two choices are possible for Yukawa interaction; i.e., so called Model I and Model II[40]. In Model I only one Higgs doublet gives masses of quarks and leptons, while one of the Higgs doublets gives masses of up-type quarks and the other does of down-type quarks and charged leptons in Model II. We assume the Model II Yukawa interaction for quarks assigning $q_{L}^{i} \rightarrow+q_{L}^{i}, u_{R}^{i} \rightarrow-u_{R}^{i}$ and $d_{R}^{i} \rightarrow+d_{R}^{i}$. Even in Model II (or Model I), FCNC can be induced at one loop level due to off-diagonal elements of the Cabibbo-Kobayasi-Maskawa (CKM) matrix, and in case due to new physics effects. Flavor non-conserving decays of Higgs bosons into quark pairs have been studied in the THDM [16, 41] and also in the context of the SUSY models[42]. We do not discuss flavor violation in the quark sector in this paper. For leptonic Yukawa interactions, the discrete symmetry is assumed to be explicitly broken, so that the LFV Yukawa couplings naturally appear in the model. Once a fundamental model is specified at high energy scales, the LFV Yukawa couplings are predicted in terms of the model parameters. In the minimal supersymmetric standard model (MSSM) slepton mixing may be the origin of LFV [12, 13, 43]. Lepton flavor violating interactions are also induced at the loop level in the Zee model where tiny neutrino masses are explained by the dynamics of the extended Higgs sector [44]. We 
here do not specify the origin of LFV, and treat the lepton flavor violating THDM as the low energy effective theory of such high energy theories.

It turns out that rare tau decay searches can give substantial upper limits on the LFV couplings of $\tau-\ell_{i}-\phi^{0}$ in the large $\tan \beta$ region, which are comparable with the values predicted by assuming some fundamental models beyond the THDM. Here $\tan \beta$ is the ratio of vacuum expectation values for neutral components of the Higgs doublets. The upper limits are rapidly relaxed for smaller $\tan \beta$ values. Therefore, under the constraint from current experimental data and the theoretical bounds, sufficiently large branching ratios of $\phi^{0} \rightarrow \tau^{ \pm} \ell_{i}^{\mp}$ are possible except for extremely large $\tan \beta$ values, which can be tested at future collider experiments. We conclude that a search for these LFV Higgs boson decays can be useful to explore the LFV couplings especially in the parameter region where rare tau decay results cannot reach. In particular, the LFV decays of the lightest Higgs boson can be one of the important probes for extended Higgs sectors when the SM-like situation would be preferred by the data at the experiments.

In Sec. III the THDM with LFV in Yukawa interaction is defined. The allowed region of the parameters of the Higgs sector is discussed by requiring the theoretical consistencies and the experimental results. In Sec. III we discuss the upper limit on the LFV Yukawa couplings in a wide range of the parameter space by using the current data for the rare tau decays. In Sec. IV LFV in Higgs boson decays is studied under the rare tau decay results. Conclusions are given in Sec. $\mathbf{\nabla}$

\section{MODEL}

In this section, the lepton flavor violating THDM is introduced, and the theoretical constraints and the experimental limits are discussed.

\section{A. Lagrangian}

We consider the Yukawa interaction for charged leptons,

$$
-\mathcal{L}_{\text {lepton }}=\bar{\ell}_{R i}\left\{Y_{\ell_{i}} \delta_{i j} \Phi_{1}+\left(Y_{\ell_{i}} \epsilon_{i j}^{L}+\epsilon_{i j}^{R} Y_{\ell_{j}}\right) \Phi_{2}\right\} \cdot L_{j}+\text { H.c. }
$$

where $\Phi_{1}$ and $\Phi_{2}$ are the scalar iso-doublets with hypercharge $1 / 2, \ell_{R i}(i=1-3)$ are singlet fields for right-handed charged leptons, $L_{i}(i=1-3)$ denote the lepton doublets and $Y_{\ell_{i}}$ are the 
Yukawa couplings for $\ell_{i}$. This interaction is reduced to be of Model II 40] in the limit $\epsilon_{i j}^{L, R} \rightarrow$ 0 with the discrete symmetry under $e_{R}^{i} \rightarrow+e_{R}^{i}, L_{i} \rightarrow+L_{i}, \Phi_{1} \rightarrow+\Phi_{1}$, and $\Phi_{2} \rightarrow-\Phi_{2}$. Nonzero values of $\epsilon_{i j}^{L, R}(i \neq j)$ yield the LFV Yukawa couplings after the diagonalization of the mass matrix. We note that in supersymmetric standard models, the Yukawa interaction for leptons is of Model II at the tree level, and $\epsilon_{i j}^{L, R}$ can be induced at the loop level due to slepton mixing 12, 13, 43]. For the quark sector, Model II Yukawa interactions are assumed to suppress FCNC, imposing the invariance under the transformation of $u_{R}^{i} \rightarrow-u_{R}^{i}$, $d_{R}^{i} \rightarrow+d_{R}^{i}, q_{L}^{i} \rightarrow+q_{L}^{i}, \Phi_{1} \rightarrow+\Phi_{1}$, and $\Phi_{2} \rightarrow-\Phi_{2}$.

The Higgs sector of the general THDM is expressed as

$$
\begin{aligned}
-\mathcal{L}_{\text {Higgs }}= & m_{1}^{2}\left|\Phi_{1}\right|^{2}+m_{2}^{2}\left|\Phi_{2}\right|^{2}-\left(m_{3}^{2} \Phi_{1}^{\dagger} \Phi_{2}+\text { H.c. }\right)+\frac{\lambda_{1}}{2}\left|\Phi_{1}\right|^{4}+\frac{\lambda_{2}}{2}\left|\Phi_{2}\right|^{4} \\
& +\lambda_{3}\left|\Phi_{1}\right|^{2}\left|\Phi_{2}\right|^{2}+\lambda_{4}\left|\Phi_{1}^{\dagger} \Phi_{2}\right|^{2}+\left\{\frac{\lambda_{5}}{2}\left(\Phi_{1}^{\dagger} \Phi_{2}\right)^{2}+\text { H.c. }\right\} \\
& +\left(\lambda_{6}\left|\Phi_{1}\right|^{2} \Phi_{1}^{\dagger} \Phi_{2}+\text { H.c. }\right)+\left(\lambda_{7}\left|\Phi_{2}\right|^{2} \Phi_{1}^{\dagger} \Phi_{2}+\text { H.c. }\right) .
\end{aligned}
$$

In Eq. (2), $m_{3}^{2}, \lambda_{5}, \lambda_{6}$ and $\lambda_{7}$ are complex in general. We here assume that all the parameters $m_{1-3}^{2}$ and $\lambda_{1-7}$ are real. The terms of $m_{3}^{2}, \lambda_{6}$ and $\lambda_{7}$ break the discrete symmetry explicitly. As we consider the model in which the discrete symmetry is explicitly broken only in the leptonic Yukawa interaction, we set the hard-breaking coupling constants to be zero in the Higgs potential; i.e., $\lambda_{6}=\lambda_{7}=0^{1}$, and retain only the soft-breaking mass parameter $m_{3}^{2}$.

There are eight degrees of freedom in the two Higgs doublet fields. Three of them are absorbed by the weak gauge bosons via the Higgs mechanism. Remaining five are physical states. After the diagonalization of the mass matrices, they correspond to two CP-even $(h$ and $H$ ), a CP-odd $(A)$, and a pair of charged $\left(H^{ \pm}\right)$Higgs bosons. The CP-even neutral states are defined such that $h$ is lighter than $H$. In our model, the eight real parameters $m_{1-3}^{2}$ and $\lambda_{1-5}$ can be described by the same number of physical parameters; i.e., the vacuum expectation value $v(\simeq 246 \mathrm{GeV})$, the Higgs boson masses $m_{h}, m_{H}, m_{A}$ and $m_{H^{ \pm}}$, the mixing angle $\alpha$ between the CP-even Higgs bosons, the ratio $\tan \beta\left(\equiv\left\langle\Phi_{2}^{0}\right\rangle /\left\langle\Phi_{1}^{0}\right\rangle\right)$ of the vacuum expectation values for two Higgs doublets, and the soft-breaking scale $M\left(\equiv \sqrt{m_{3}^{2} / \sin \beta \cos \beta}\right)$ for the discrete symmetry. The quartic couplings are expressed in terms of physical parameters

\footnotetext{
${ }^{1}$ Even in such a case, $\lambda_{6}$ and $\lambda_{7}$ are effectively induced at the loop level. They are suppressed by the loop factor, so that we here neglect these small effects.
} 
by 45$]$

$$
\begin{aligned}
& \lambda_{1}=\frac{1}{v^{2} \cos ^{2} \beta}\left(-\sin ^{2} \beta M^{2}+\sin ^{2} \alpha m_{h}^{2}+\cos ^{2} \alpha m_{H}^{2}\right), \\
& \lambda_{2}=\frac{1}{v^{2} \sin ^{2} \beta}\left(-\cos ^{2} \beta M^{2}+\cos ^{2} \alpha m_{h}^{2}+\sin ^{2} \alpha m_{H}^{2}\right), \\
& \lambda_{3}=\frac{1}{v^{2}}\left\{-M^{2}+2 m_{H^{ \pm}}^{2}+\frac{\sin 2 \alpha}{\sin 2 \beta}\left(m_{H}^{2}-m_{h}^{2}\right)\right\}, \\
& \lambda_{4}=\frac{1}{v^{2}}\left(M^{2}+m_{A}^{2}-2 m_{H^{ \pm}}^{2}\right), \\
& \lambda_{5}=\frac{1}{v^{2}}\left(M^{2}-m_{A}^{2}\right) .
\end{aligned}
$$

The relative size of the parameter $M$ against $v$ determines the decoupling property of the Higgs sector. For the case of $M^{2} \gg v^{2}, m_{H}, m_{A}$ and $m_{H^{ \pm}}$turn out to be around $M$. They decouple from low energy observables by a factor of $v^{2} / M^{2}$, and the lightest Higgs boson $h$ becomes the SM-like one[45, 46]. The THDM effectively becomes the SM at low energies. On the contrary, when $M^{2} \lesssim v^{2}$, the Higgs boson masses can be varied by the variation of relative size of quartic couplings. In such a case, the parameter space is strongly constrained by the conditions from perturbative unitarity, which we shall discuss in the next subsection. Notice that even for $M^{2} \lesssim v^{2}$ we can define the "SM-like" limit by setting $\sin (\alpha-\beta) \sim-1$ [45, 47]. The coupling constants of $h V V\left(V V=W^{+} W^{-}\right.$and $\left.Z Z\right)$ then coincide with those in the SM at the tree level, and there are no $H V V$ couplings.

\section{B. Constraint on the Higgs parameters}

Parameters of the Higgs sector are constrained from requirements of theoretical consistencies and also from the current experimental results. We here take into account two kinds

of theoretical conditions; i.e., vacuum stability [31] and perturbative unitarity [32, 33, 34] at the tree level. The condition for vacuum stability is expressed by

$$
\lambda_{1}>0, \quad \lambda_{2}>0, \quad \sqrt{\lambda_{1} \lambda_{2}}+\lambda_{3}+\operatorname{MIN}\left[0, \lambda_{4}+\lambda_{5}, \lambda_{4}-\lambda_{5}\right]>0,
$$

and that for tree-level unitarity is described as

$$
\left|\left\langle\phi_{3} \phi_{4}\left|a^{0}\right| \phi_{1} \phi_{2}\right\rangle\right|<\xi
$$

where $\left\langle\phi_{3} \phi_{4}\left|a^{0}\right| \phi_{1} \phi_{2}\right\rangle$ is the $s$-wave amplitude for the process of $\phi_{1} \phi_{2} \rightarrow \phi_{3} \phi_{4}$ with $\phi_{a}(a=1$ 4) denoting Higgs bosons and longitudinal components of weak gauge bosons. We employ 
the conditions given in Refs. [33, 45] in which the fourteen channels are taken into account in the THDM; i.e., $Z Z, h Z, H Z, A Z, h h, H H, A A, h H, h A, H A, W^{+} W^{-}, H^{+} H^{-}$and $W^{ \pm} H^{\mp}$. We take the criterion $\xi$ to be $1[32]$ (and also 1/2[40] for comparison). For the top and bottom Yukawa couplings with Higgs bosons $\left(y_{t \bar{t} \phi^{0}}\right.$ and $\left.y_{b \bar{b} \phi^{0}}\right)$, we just put the criterion,

$$
\left|y_{q \bar{q} \phi^{0}}\right|^{2}<4 \pi, \quad(q=t, b)
$$

For example, this condition on $y_{t \bar{t} A}$ and $y_{b \bar{b} A}$ gives upper and lower limits for $\tan \beta(0.2 \lesssim$ $\tan \beta \lesssim 100-200)^{2}$. As shown in the next section, the conditions of Eqs. (8) and (9) give more strict upper bound on $\tan \beta$ than that of Eq. (10) in a wide parameter region.

Next we consider the experimental constraints, which are provided by the LEP precision data [35], the $b \rightarrow s \gamma$ results [37], and the direct search results for the Higgs bosons [35]. The LEP precision data provide the strong constraint on the new physics structure via the gauge-boson two-point functions. The constraint on $\rho$ parameter indicates that the Higgs sector is approximately custodial $S U(2)$ symmetric. This requirement is satisfied when (i) $m_{H^{ \pm}} \simeq m_{A}$, (ii) $m_{H^{ \pm}} \simeq m_{H}$ with $\sin ^{2}(\alpha-\beta) \simeq 1$, and (iii) $m_{H^{ \pm}} \simeq m_{h}$ with $\cos ^{2}(\alpha-\beta) \simeq 1$ [36]. It is known that in Model II, the $b \rightarrow s \gamma$ result gives the lower bound on the charged Higgs boson mass [37, 38]. We here take into account this bound by requiring $m_{H^{ \pm}} \gtrsim 350 \mathrm{GeV}$.

\section{LFV parameters $\kappa_{i j}^{L, R}$}

The tau lepton associated LFV interactions in Eq. (10) can be reduced in the mass eigenbasis of each field to

$$
\begin{aligned}
-\mathcal{L}_{\tau \mathrm{LFV}}= & \frac{m_{\tau}}{v \cos ^{2} \beta}\left(\kappa_{3 i}^{L} \bar{\tau} \mathrm{P}_{L} \ell_{i}+\kappa_{i 3}^{R} \bar{\ell}_{i} \mathrm{P}_{L} \tau\right)\{\cos (\alpha-\beta) h+\sin (\alpha-\beta) H-\mathrm{i} A\} \\
& +\frac{\sqrt{2} m_{\tau}}{v \cos ^{2} \beta}\left(\kappa_{3 i}^{L} \bar{\tau} \mathrm{P}_{L} \nu_{i}+\kappa_{i 3}^{R} \bar{\ell}_{i} \mathrm{P}_{L} \nu_{\tau}\right) H^{-}+\text {H.c. }
\end{aligned}
$$

where $\mathrm{P}_{L}$ is the projection operator to the left-handed field, and $\ell_{1}$ and $\ell_{2}$ respectively represent $e$ and $\mu$. In general, the LFV parameters $\kappa_{i j}^{L, R}$ can be expressed in terms of $\epsilon_{i j}^{L, R}$ and

\footnotetext{
${ }^{2}$ Similar bounds are obtained for example in Ref. [48].
} 
$\tan \beta .^{3}$ We here take these $\kappa_{i j}^{L, R}$ as effective couplings, and investigate their phenomenological consequences. We note that Eq. (III) is exact in the limit of $m_{\ell_{i}} \rightarrow 0$. The terms of $\kappa_{i 3}^{L}$ and $\kappa_{3 i}^{R}(i=1,2)$ are proportional to $m_{\ell_{i}}$, so that they decouple in this approximation.

We briefly discuss relationship between $\kappa_{i j}^{L, R}$ and new physics models beyond the cut-off scale of the effective THDM. When a new physics model is specified at the high energy scale, $\kappa_{i j}^{L, R}$ can be predicted as a function of the model parameters. For example, in the MSSM, slepton mixing can be a source of LFV. Notice that the induced LFV Higgs interactions do not necessarily decouple in the limit where the SUSY particles are sufficiently heavy, because their couplings only depend on the ratio of the SUSY parameters. Therefore, the Higgs associated LFV processes can become important in a scenario with the soft-SUSYbreaking scale $m_{\text {SusY }}$ to be much higher than the electroweak one. In the MSSM, predicted values of $\left|\kappa_{3 i}^{L}\right|^{2}$ can be as large as of $\mathcal{O}\left(10^{-6}\right)$ when $m_{\text {SUSY }}$ is a few TeV 15, 27]. In the MSSM with right-handed neutrinos, slepton mixing may be a consequence of running effects of the neutrino Yukawa couplings between the scale of the grand unification and that of the right-handed neutrinos 43 . The parameters $\kappa_{3 i}^{L}$ are mainly induced by mixing of left-handed sleptons 12, 13, 14, 15, 25, 27]. The LFV Yukawa interactions can also appear effectively in the Zee model[44]. The LFV parameters $\kappa_{i j}^{L, R}$ are induced through flavor violating couplings in the charged scalar interactions with leptons.

\section{BOUND ON LFV YUKAWA COUPLINGS FROM RARE TAU DECAYS}

In order to constrain the LFV parameters $\left|\kappa_{3 i}^{L}\right|$ and $\left|\kappa_{i 3}^{R}\right|$, we take into account the data for rare tau decay processes such as $\tau \rightarrow \ell_{i} P^{0}, \tau \rightarrow \ell_{i} M^{+} M^{\prime-}, \tau \rightarrow \ell_{i} \ell^{\prime+} \ell^{\prime-}$, and $\tau \rightarrow \ell_{i} \gamma$, where $P^{0}$ represents $\pi^{0}, \eta$ and $\eta^{\prime}$ mesons, $M^{ \pm}\left(M^{ \pm}\right)$does $\pi^{ \pm}$and $K^{ \pm}$mesons, and $\ell^{\prime \pm}=$ $e^{ \pm}$and $\mu^{ \pm}$. The list of the current data from the B factories are summarized in Table प 2. 3, 3, 4, 5, 6, 6, 8, 9]. These bounds may be improved at the super B factory around a digit [21]. In our analysis, we take the underlined data in Table पas our numerical inputs.

$\overline{{ }^{3} \mathrm{LFV}}$ parameters $\kappa_{i j}^{L, R}$ can be expressed as

$$
\kappa_{i j}^{X}=-\frac{\epsilon_{i j}^{X}}{\left\{1+\left(\epsilon_{33}^{L}+\epsilon_{33}^{R}\right) \tan \beta\right\}^{2}} \quad(X=L, R),
$$

for the case of $\epsilon_{i j}^{L, R} \tan \beta \ll \mathcal{O}(1)$ which is satisfied in the MSSM case 12, 13. 


\begin{tabular}{|c|c|c|}
\hline Mode & Belle (90\% CL) & $\operatorname{BaBar}(90 \% \mathrm{CL})$ \\
\hline$\tau^{-} \rightarrow e^{-} \pi^{0}$ & $\underline{1.9 \times 10^{-7}[2]}$ & \\
\hline$\tau^{-} \rightarrow e^{-} \eta$ & $\underline{2.4 \times 10^{-7}[2]}$ & \\
\hline$\tau^{-} \rightarrow e^{-} \eta^{\prime}$ & $\underline{10 \times 10^{-7}[2]}$ & \\
\hline$\tau^{-} \rightarrow \mu^{-} \pi^{0}$ & $\underline{4.1 \times 10^{-7}}[2]$ & \\
\hline$\tau^{-} \rightarrow \mu^{-} \eta$ & $\underline{1.5 \times 10^{-7}}[2]$ & \\
\hline$\tau^{-} \rightarrow \mu^{-} \eta^{\prime}$ & $\underline{4.7 \times 10^{-7}}[2]$ & \\
\hline$\tau^{-} \rightarrow e^{-} \pi^{+} \pi^{-}$ & $8.4 \times 10^{-7}[3]$ & $1.2 \times 10^{-7}[4]$ \\
\hline$\tau^{-} \rightarrow e^{-} \pi^{+} K^{-}$ & $5.7 \times 10^{-7}[3]$ & $\underline{3.2 \times 10^{-7}[4]}$ \\
\hline$\tau^{-} \rightarrow e^{-} K^{+} \pi^{-}$ & $5.6 \times 10^{-7}[3]$ & $\underline{1.7 \times 10^{-7}[4]}$ \\
\hline$\tau^{-} \rightarrow e^{-} K^{+} K^{-}$ & $3.0 \times 10^{-7}[3]$ & $\underline{1.4 \times 10^{-7}}[4]$ \\
\hline$\tau^{-} \rightarrow \mu^{-} \pi^{+} \pi^{-}$ & $\underline{2.8 \times 10^{-7}}[3]$ & $2.9 \times 10^{-7}[4]$ \\
\hline$\tau^{-} \rightarrow \mu^{-} \pi^{+} K^{-}$ & $6.3 \times 10^{-7}[3]$ & $\underline{2.6 \times 10^{-7}}[4]$ \\
\hline$\tau^{-} \rightarrow \mu^{-} K^{+} \pi^{-}$ & $15.5 \times 10^{-7}[3]$ & $\underline{3.2 \times 10^{-7}}[4]$ \\
\hline$\tau^{-} \rightarrow \mu^{-} K^{+} K^{-}$ & $11.7 \times 10^{-7}[3]$ & $\underline{2.5 \times 10^{-7}[4]}$ \\
\hline$\tau^{-} \rightarrow e^{-} e^{+} e^{-}$ & $3.5 \times 10^{-7}[5]$ & $\underline{2.0 \times 10^{-7}[6]}$ \\
\hline$\tau^{-} \rightarrow e^{-} \mu^{+} \mu^{-}$ & $\underline{2.0 \times 10^{-7}}[5]$ & $3.3 \times 10^{-7}[6]$ \\
\hline$\tau^{-} \rightarrow \mu^{-} e^{+} e^{-}$ & $\underline{1.9 \times 10^{-7}}[5]$ & $2.7 \times 10^{-7}[6]$ \\
\hline$\tau^{-} \rightarrow \mu^{-} \mu^{+} \mu^{-}$ & $2.0 \times 10^{-7}[5]$ & $\underline{1.9 \times 10^{-7}}[\underline{6}]$ \\
\hline$\tau \rightarrow e \gamma$ & $\underline{3.9 \times 10^{-7}}[7]$ & \\
\hline$\tau \rightarrow \mu \gamma$ & $3.1 \times 10^{-7}[8]$ & $\underline{6.8 \times 10^{-8}}[9]$ \\
\hline
\end{tabular}

TABLE I: Current experimental limits on branching ratios of the LFV rare tau decays.

The branching ratios for these $\tau^{-}$decays are calculated in our model as

$$
\begin{aligned}
& \operatorname{Br}\left(\tau^{-} \rightarrow \ell_{i}^{-} \pi^{0}\right)=\frac{3 G_{F}^{2} m_{\tau}^{3} m_{\pi^{0}}^{4} F_{\pi}^{2} \tau_{\tau}\left|\kappa_{3 i}\right|^{2}}{32 \pi \cos ^{4} \beta}\left(1-\frac{m_{\pi^{0}}^{2}}{m_{\tau}^{2}}\right)^{2}\left(\frac{m_{u} \cot \beta-m_{d} \tan \beta}{m_{u}+m_{d}}\right)^{2} \frac{1}{m_{A}^{4}}, \\
& \operatorname{Br}\left(\tau^{-} \rightarrow \ell_{i}^{-} \eta\right)=\frac{9 G_{F}^{2} m_{\tau}^{3} m_{\eta}^{4} F_{\eta}^{2} \tau_{\tau}\left|\kappa_{3 i}\right|^{2}}{32 \pi \cos ^{4} \beta}\left(1-\frac{m_{\eta}^{2}}{m_{\tau}^{2}}\right)^{2}\left(\frac{m_{u} \cot \beta+\left(m_{d}-2 m_{s}\right) \tan \beta}{m_{u}+m_{d}+4 m_{s}}\right)^{2} \frac{1}{m_{A}^{4}}, \\
& \operatorname{Br}\left(\tau^{-} \rightarrow \ell_{i}^{-} \pi^{+} \pi^{-}\right)=\frac{G_{F}^{2} m_{\tau}^{5} B_{0}^{2} \tau_{\tau}\left|\kappa_{3 i}\right|^{2}}{256 \pi^{3} \cos ^{6} \beta}\left\{m_{d}\left(\frac{\sin \alpha \cos (\alpha-\beta)}{m_{h}^{2}}-\frac{\cos \alpha \sin (\alpha-\beta)}{m_{H}^{2}}\right)\right.
\end{aligned}
$$




$$
\begin{aligned}
& \left.-m_{u} \cot \beta\left(\frac{\cos \alpha \cos (\alpha-\beta)}{m_{h}^{2}}+\frac{\sin \alpha \sin (\alpha-\beta)}{m_{H}^{2}}\right)\right\}^{2} \\
& \operatorname{Br}\left(\tau^{-} \rightarrow \ell_{i}^{-} K^{+} K^{-}\right)=\frac{G_{F}^{2} m_{\tau}^{5} B_{0}^{2} \tau_{\tau}\left|\kappa_{3 i}\right|^{2}}{256 \pi^{3} \cos ^{6} \beta}\left\{m_{s}\left(\frac{\sin \alpha \cos (\alpha-\beta)}{m_{h}^{2}}-\frac{\cos \alpha \sin (\alpha-\beta)}{m_{H}^{2}}\right)\right. \\
& \left.-m_{u} \cot \beta\left(\frac{\cos \alpha \cos (\alpha-\beta)}{m_{h}^{2}}+\frac{\sin \alpha \sin (\alpha-\beta)}{m_{H}^{2}}\right)\right\}^{2} \\
& \operatorname{Br}\left(\tau^{-} \rightarrow \ell_{i}^{-} \pi^{+} K^{-}\right)=\operatorname{Br}\left(\tau^{-} \rightarrow \ell_{i}^{-} K^{+} \pi^{-}\right) \sim \frac{G_{F}^{2} m_{W}^{4}}{32 \pi^{4}}\left|V_{u d} V_{u s}^{*}\right|^{2} \operatorname{Br}\left(\tau^{-} \rightarrow \ell_{i}^{-} K^{+} K^{-}\right), \\
& \operatorname{Br}\left(\tau^{-} \rightarrow \ell_{i}^{-} \ell^{\prime+} \ell^{\prime-}\right) \\
& =\frac{G_{F}^{2} m_{\ell^{\prime}}^{2} m_{\tau}^{7} \tau_{\tau}\left|\kappa_{3 i}\right|^{2}}{1536 \pi^{3} \cos ^{6} \beta}\left\{\left(\frac{\sin \alpha \cos (\alpha-\beta)}{m_{h}^{2}}-\frac{\cos \alpha \sin (\alpha-\beta)}{m_{H}^{2}}\right)^{2}+\frac{\sin ^{2} \beta}{m_{A}^{4}}\right\}, \\
& \operatorname{Br}\left(\tau^{-} \rightarrow \ell_{i}^{-} \ell_{i}^{+} \ell_{i}^{-}\right) \\
& =\frac{G_{F}^{2} m_{\ell_{i}}^{2} m_{\tau}^{7} \tau_{\tau}\left|\kappa_{3 i}\right|^{2}}{2048 \pi^{3} \cos ^{6} \beta}\left[\left\{\left(\frac{\sin \alpha \cos (\alpha-\beta)}{m_{h}^{2}}-\frac{\cos \alpha \sin (\alpha-\beta)}{m_{H}^{2}}\right)^{2}+\frac{\sin ^{2} \beta}{m_{A}^{4}}\right\}\right. \\
& \left.+\frac{2}{3}\left(\frac{\sin \alpha \cos (\alpha-\beta)}{m_{h}^{2}}-\frac{\cos \alpha \sin (\alpha-\beta)}{m_{H}^{2}}\right) \frac{\sin \beta}{m_{A}^{2}}\right] \text {, } \\
& \operatorname{Br}\left(\tau^{-} \rightarrow \ell_{i}^{-} \gamma\right)=\frac{\alpha_{\mathrm{em}} G_{F}^{2} m_{\tau}^{9} \tau_{\tau}}{72(4 \pi)^{4} \cos ^{6} \beta} \\
& \times\left\{\left|\kappa_{3 i}^{L}\right|^{2}\left(\frac{\sin \alpha \cos (\alpha-\beta)}{m_{h}^{2}} f_{-}\left(x_{h}\right)-\frac{\cos \alpha \sin (\alpha-\beta)}{m_{H}^{2}} f_{-}\left(x_{H}\right)+\frac{\sin \beta}{m_{A}^{2}} f_{+}\left(x_{A}\right)\right)^{2}\right. \\
& \left.+\left|\kappa_{i 3}^{R}\right|^{2}\left(\frac{\sin \alpha \cos (\alpha-\beta)}{m_{h}^{2}} f_{-}\left(x_{h}\right)-\frac{\cos \alpha \sin (\alpha-\beta)}{m_{H}^{2}} f_{-}\left(x_{H}\right)+\frac{\sin \beta}{m_{A}^{2}} f_{+}\left(x_{A}\right)+\frac{\sin \beta}{m_{H^{ \pm}}^{2}}\right)^{2}\right\},
\end{aligned}
$$

where $\left|\kappa_{3 i}\right|^{2} \equiv\left|\kappa_{3 i}^{L}\right|^{2}+\left|\kappa_{i 3}^{R}\right|^{2}, G_{F}$ is the Fermi constant, $F_{\pi}$ and $F_{\eta}$ are the decay constants of the pion and the $\eta$ meson which are defined as shown in Ref.[19], $\tau_{\tau}$ is the life time of the tau lepton, and $\alpha_{\mathrm{em}}$ is the fine structure constant. The expression in Eqs. (14) is deduced in the approximation of non-mixing between the octet and singlet states; i.e., $\eta=\eta_{8}$. In Eqs. (15) and (16), $B_{0}$ is the matrix element for a pair production of pions, which is evaluated at the leading order as $B_{0}=m_{\pi^{ \pm}}^{2} /\left(m_{u}+m_{d}\right)\left(=m_{K^{ \pm}}^{2} /\left(m_{u}+m_{s}\right)\right)$ by using the chiral Lagrangian[19]. In Eq. (18), $\ell^{\prime}$ represents either $e$ or $\mu$ but $\ell^{\prime} \neq \ell_{i}$. In Eq. (201), $f_{ \pm}(x) \equiv 1 \pm 3(3+2 \ln x)$ and $x_{\phi^{0}} \equiv m_{\tau}^{2} / m_{\phi^{0}}^{2}\left(\phi^{0}=h, H \text { and } A\right)^{4}$. We here omit the sub-leading contributions such as $\mathcal{O}\left(m_{\ell_{i}} / m_{\tau}\right)^{5}$. We also do not consider the contribution to

\footnotetext{
${ }^{4}$ In this calculation, masses of neutrinos are ignored.

5 The crossing terms between $\kappa_{3 i}^{L}$ and $\kappa_{i 3}^{R}$ do not appear in this approximation.
} 
$\tau \rightarrow \ell_{i} P^{0}, \tau \rightarrow \ell_{i} \ell^{\prime+} \ell^{\prime-}$ and $\tau \rightarrow \ell_{i} M^{+} M^{\prime-}$ from gauge boson mediated LFV diagrams which are induced at the loop level. In the SUSY-like limit $\left(m_{H} \simeq m_{A}\right)$, Eq. (19) coincides with the results of Ref. 13$]^{6}$, and the ratio between Eq.(14) and Eq. (19) reproduces the result shown in Ref. 14]. Our result of Eq. (18) is consistent with Ref. 19], and Eqs. (13), (14), (15), and (16) are also consistent with the results in Ref. 19] up to the colour factor ${ }^{7}$. We keep the terms with $\mathcal{O}\left(m_{P^{0}}^{2} / m_{\tau}^{2}\right)$ in Eqs. (13) and (14) although we ignore those with $\mathcal{O}\left(m_{M}^{2} / m_{\tau}^{2}\right)$ in the derivation of Eqs. (15) and (16). The expression of Eq. (20) is compatible with Ref. 18]. The branching ratios of the $\tau^{+}$decay are the same as those of $\tau^{-}$in Eqs. (13)-(20).

Some comments are in order. First, the processes $\tau \rightarrow \ell_{i} P^{0}\left(P^{0}=\pi^{0}, \eta\right.$ and $\left.\eta^{\prime}\right)$ are mediated only by the CP-odd Higgs boson $A$, and their branching ratios strongly depend on masses of the outgoing mesons. Because of the cancellation between the singlet and octet component in the $\eta^{\prime}$ meson, the bound on $\left|\kappa_{3 i}\right|^{2}$ from $\tau \rightarrow \ell_{i} \eta^{\prime}$ may be less important than that from $\tau \rightarrow \ell_{i} \eta[15]$. We do not include $\tau \rightarrow \ell_{i} \eta^{\prime}$ in our analysis below. From Table $\llbracket$ and Eqs. (13)-(14), it is expected that $\tau \rightarrow \ell_{i} \eta$ gives the most stringent upper limit on $\left|\kappa_{3 i}\right|^{2}$ of these three modes. Second, the expressions for the branching ratios of $\tau \rightarrow \ell_{i} M^{+} M^{-}$with $M^{ \pm}=\pi^{ \pm}$and $K^{ \pm}$are the same up to the difference of down type quarks $d$ and $s$ as seen in Eqs. (15) and (16). These modes are mediated by the CP-even Higgs bosons $h$ and $H$. As the mass of the $s$ quark is much greater than that of the $d$ quark while the experimental upper limits on them are the same order, $\tau \rightarrow \ell_{i} K^{+} K^{-}$gives the more stringent bounds on $\left|\kappa_{3 i}\right|^{2}$ than $\tau \rightarrow \ell_{i} \pi^{+} \pi^{-}$in a wide region of the parameter space. It is also found that $\tau \rightarrow \ell_{i} \pi^{ \pm} K^{\mp}$ cannot contribute to limit values of $\left|\kappa_{3 i}\right|^{2}$. Their branching ratios are one-loop induced and much smaller than those of $\tau \rightarrow \ell_{i}^{-} K^{+} K^{-}$due to the loop suppression factor and the CKM factor $\left|V_{u d} V_{u s}^{*}\right|^{2}$, while the data for these processes are the same order: see Eq. (17). Third, in Eqs. (18) and (19) the branching ratios of $\tau^{-} \rightarrow \ell_{i}^{-} \ell^{\prime+} \ell^{\prime-}$ are proportional to the mass of $\ell^{\prime}$. As the data for $\tau^{-} \rightarrow \ell_{i}^{-} \mu^{+} \mu^{-}$and $\tau^{-} \rightarrow \ell_{i}^{-} e^{+} e^{-}$are the same order, $\tau^{-} \rightarrow \ell_{i}^{-} \mu^{+} \mu^{-}$ provide much stronger upper limits on $\left|\kappa_{3 i}\right|^{2}$ within the tree approximation because of the mass difference between electrons and muons ${ }^{8}$. The modes $\tau^{-} \rightarrow \ell_{i}^{-} \ell^{\prime+} \ell^{\prime-}$ are mediated by all neutral Higgs bosons $h, H$ and $A$. Finally, $\tau^{-} \rightarrow \ell_{i}^{-} \gamma$ are one-loop induced processes in

\footnotetext{
${ }^{6}$ Eq. (19) is also consistent with the expression in Ref. 22 .

7 The color factor is missing in the expressions in Ref. [19].

8 At the loop level, the branching ratio of the $\tau^{-} \rightarrow \ell_{i}^{-} e^{+} e^{-}$process can become larger than that of $\tau^{-} \rightarrow \ell_{i}^{-} \mu^{+} \mu^{-}$because of the phase space dependence in the photonic penguin diagram [23].
} 
our model. All kinds of the Higgs bosons are included in the one-loop diagrams. Notice that as shown in Eq. (20) the one loop diagram with a charged Higgs boson is asymmetric with respect to chirality of the tau lepton, as the neutrino in the diagram is left-handed. In the following, we study the bound on $\left|\kappa_{3 i}\right|^{2}$, and do not treat $\left|\kappa_{3 i}^{L}\right|^{2}$ and $\left|\kappa_{i 3}^{R}\right|^{2}$ separately.

Since branching ratios for $\tau^{-} \rightarrow \ell_{i}^{-} P^{0}, \tau^{-} \rightarrow \ell_{i}^{-} M^{+} M^{-}, \tau^{-} \rightarrow \ell_{i}^{-} \ell^{\prime+} \ell^{\prime-}$ and $\tau^{-} \rightarrow \ell_{i}^{-} \gamma$ depend on different combinations of the Higgs boson masses, independent information can be obtained for the model parameters by measuring each of them. When all the masses of Higgs bosons are large, these decay processes decouple by a factor of $1 / m_{\text {Higgs }}^{4}$. Although these branching ratios are complicated functions of the mixing angles, each of them can be simply expressed to be proportional to $\tan ^{6} \beta$ for $\tan \beta \gg 1$ in the SM-like region $(\sin (\alpha-\beta) \sim-1)$. This $\tan ^{6} \beta$ dependence is a common feature of the tau-associated LFV processes with the Higgs-mediated 4-Fermi interactions ${ }^{9}$.

The experimental upper limit on $\left|\kappa_{3 i}\right|^{2}$ can be obtained by using the experimental results given in Table I and analytic expressions for the decay branching ratios in Eqs. (13)-(20). For instance, let us consider the bound from the $\tau \rightarrow \mu \eta$ results [14]. From Eq. (14) we obtain

$$
\left|\kappa_{32}\right|^{2} \leq\left(\left|\kappa_{32}^{\max }\right|^{2}\right)_{\tau \rightarrow \mu \eta} \equiv \frac{128 \pi \operatorname{Br}(\tau \rightarrow \mu \eta)_{\exp } m_{A}^{4}}{9 G_{F}^{2} m_{\tau}^{3} m_{\eta}^{4} F_{\eta}^{2} \tau_{\tau}\left(1-\frac{m_{\eta}^{2}}{m_{\tau}^{2}}\right)^{2}} \frac{\cos ^{6} \beta}{\sin ^{2} \beta}
$$

where $\operatorname{Br}(\tau \rightarrow \mu \eta)_{\exp }$ is the experimental upper limit on the branching ratio of $\tau \rightarrow \mu \eta$ in Table 【. In particular, for $\tan \beta \gg 1$, the right-hand-side can be expressed by

$$
\left(\left|\kappa_{32}^{\max }\right|^{2}\right)_{\tau \rightarrow \mu \eta} \simeq 2.3 \times 10^{-4} \times\left(\frac{m_{A}}{350[\mathrm{GeV}]}\right)^{4}\left(\frac{30}{\tan \beta}\right)^{6} .
$$

It can be easily seen that the bound $\left(\left|\kappa_{32}^{\max }\right|^{2}\right)_{\tau \rightarrow \mu \eta}$ is rapidly relaxed in the region with small $\tan \beta$ and large $m_{A}$. In a similar way to Eq. (21), the maximal allowed value $\left(\left|\kappa_{3 i}^{\max }\right|^{2}\right)_{\text {mode }}$ can be calculated for each mode. The combined upper limit $\left(\left|\kappa_{3 i}^{\max }\right|^{2}\right)$ is then given by

$$
\left|\kappa_{3 i}^{\max }\right|^{2} \equiv \operatorname{MIN}\left\{\left(\left|\kappa_{3 i}^{\max }\right|^{2}\right)_{\tau \rightarrow \ell \eta},\left(\left|\kappa_{3 i}^{\max }\right|^{2}\right)_{\tau \rightarrow \ell \mu^{+} \mu^{-}},\left(\left|\kappa_{3 i}^{\max }\right|^{2}\right)_{\tau \rightarrow \ell K^{+} K^{-}},\left(\left|\kappa_{3 i}^{\max }\right|^{2}\right)_{\tau \rightarrow \ell \gamma}, \cdots\right\}
$$

\footnotetext{
9 This also applies to the one-loop induced $\tau \rightarrow \ell_{i} \gamma$ processes whose diagrams have the same tan $\beta$ dependence.
} 


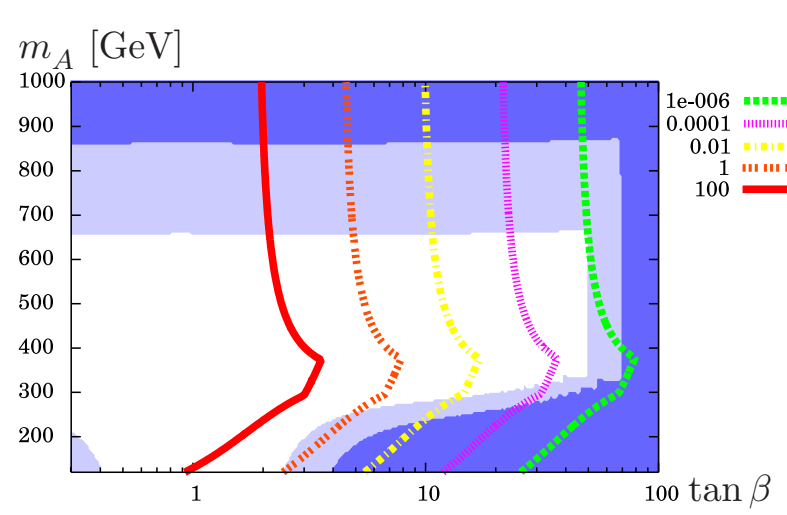

(a)

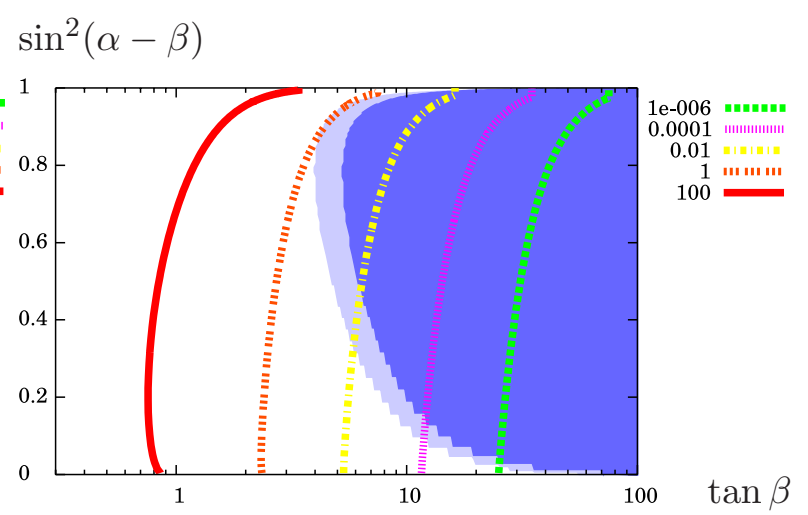

(b)

FIG. 1: Contours of $\left|\kappa_{32}^{\max }\right|^{2}$, the possible maximal value of $\left|\kappa_{32}\right|^{2}$ from the rare tau decay results, are shown (a) in the $\tan \beta-m_{A}$ plane and (b) in the $\tan \beta-\sin ^{2}(\alpha-\beta)$ plane. The parameters are taken to be (a) $m_{h}=120 \mathrm{GeV}, m_{H}=m_{H^{ \pm}}=350 \mathrm{GeV}$ and $\sin (\alpha-\beta)=-0.9999$, and (b) $m_{h}=120 \mathrm{GeV}$ and $m_{H}=m_{A}=m_{H^{ \pm}}=350 \mathrm{GeV}$. The remaining parameter $M$ is scanned from 0 to $1,000 \mathrm{GeV}$. The dark (light) shaded area indicates the excluded region by the theoretical requirements of Eqs. (8), (9) and (10) with $\xi=1(\xi=1 / 2)$.

As shown below, $\tau \rightarrow \ell_{i} \eta$ and $\tau \rightarrow \ell_{i} \gamma$ give the strongest upper limits on $\left|\kappa_{3 i}\right|^{2}$ in a wide range of the parameter space. In addition, in some parameter regions $\tau \rightarrow \ell_{i} K^{+} K^{-}$and $\tau \rightarrow \ell_{i} \mu^{+} \mu^{-}$can also give similar limits on $\left|\kappa_{3 i}\right|^{2}$ to those from the above two processes.

In Figs. 11(a) and 가(b), contour plots for $\left|\kappa_{32}^{\max }\right|^{2}$ are shown under the rare tau decay results in the $\tan \beta-m_{A}$ plane and the $\tan \beta-\sin ^{2}(\alpha-\beta)$ plane, respectively. The combined excluded region from the theoretical conditions in Eqs. (8), (9) and (10) is indicated by the dark shaded area for the criterion $\xi=1$ and by the light one for $\xi=1 / 2$. In Fig. 1ㄱ(a), parameters of the Higgs sector are taken to be $m_{h}=120 \mathrm{GeV}, m_{H}=m_{H^{ \pm}}=350 \mathrm{GeV}$ and $\sin (\alpha-\beta)=-0.9999$. In Fig. 10 (b), those are $m_{h}=120 \mathrm{GeV}$ and $m_{H}=m_{A}=m_{H^{ \pm}}=350$ $\mathrm{GeV}$. The value of $\left|\kappa_{32}^{\max }\right|^{2}$ is independent of $M$, the soft-breaking scale of the discrete symmetry. On the other hand, theoretical bounds from vacuum stability and perturbative unitarity are sensitive to $M$. Therefore, we evaluate such a theoretical allowed region by scanning $M$ to be from 0 to $1000 \mathrm{GeV}$. We also take into account the constraint from the $\rho$ parameter measurement and the $b \rightarrow s \gamma$ result by taking $\sin (\alpha-\beta) \simeq-1$ and $m_{H}=m_{H^{ \pm}}$ with $m_{H^{ \pm}} \gtrsim 350 \mathrm{GeV}$ for Fig 1-(a), and $m_{A}=m_{H^{ \pm}}$with $m_{H^{ \pm}} \gtrsim 350 \mathrm{GeV}$ for Fig 1)(b). From the both figures, it is easily found that the value of $\left|\kappa_{32}^{\max }\right|^{2}$ can extensively be larger for 


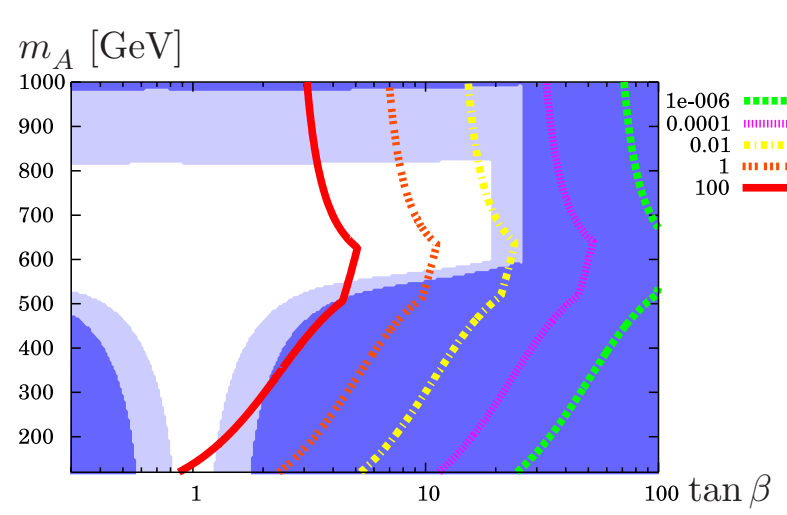

(a)

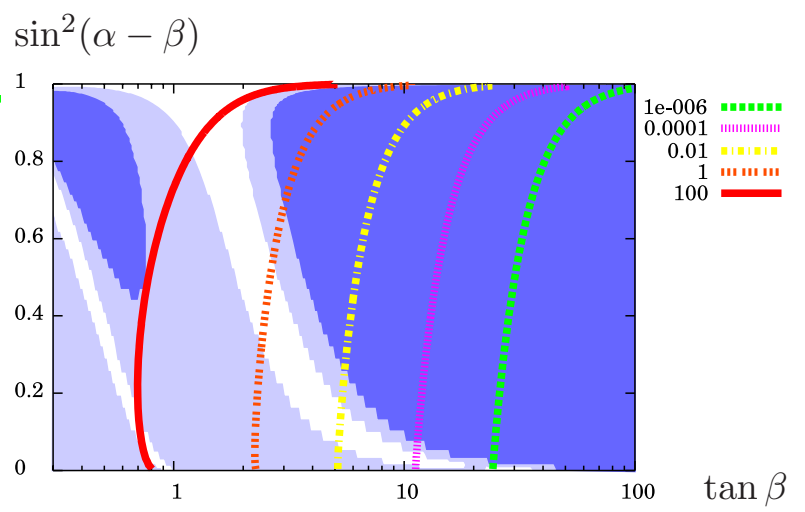

(b)

FIG. 2: Contour plots of $\left|\kappa_{32}^{\max }\right|^{2}$ are shown (a) in the $\tan \beta-m_{A}$ plane and (b) in the $\tan \beta-\sin ^{2}(\alpha-\beta)$ plane similar to Figs. ㅍ. (a) and ㄱ. (b) but with different parameter choices; (a) $m_{h}=120 \mathrm{GeV}$, $m_{H}=m_{H^{ \pm}}=600 \mathrm{GeV}$ and $\sin (\alpha-\beta)=-0.9999$, and (b) $m_{h}=120 \mathrm{GeV}$ and $m_{H}=m_{A}=$ $m_{H^{ \pm}}=600 \mathrm{GeV}$. The dark (light) shaded area indicates the excluded region by the theoretical requirements of Eqs. (8), (9) and (10) with $\xi=1(\xi=1 / 2)$.

smaller $\tan \beta$ in the allowed region under the theoretical constraints. For $\tan \beta \lesssim 10(30)$, $\left|\kappa_{32}^{\max }\right|^{2}$ can be $\mathcal{O}(0.1)\left(\mathcal{O}\left(10^{-4}\right)\right)$. Among the rare tau decay processes, $\tau \rightarrow \mu \eta$ and $\tau \rightarrow \mu \gamma$ provide the most stringent constraints on $\left|\kappa_{32}\right|^{2}$. While $\tau \rightarrow \mu \eta$ is mediated only by $A$, $\tau \rightarrow \mu \gamma$ depends on the masses of $h, H, A$ and $H^{ \pm}$. For $\sin ^{2}(\alpha-\beta) \sim 1$ and $m_{A} \sim m_{H}$, the branching ratio of $\tau \rightarrow \mu \gamma$ in Eq. (20) is suppressed because of the cancellation between the one-loop diagrams of $A$ and $H$. Therefore, $\left|\kappa_{32}\right|^{2}$ is bounded most strongly by the $\tau \rightarrow \mu \eta$ result for this case ${ }^{10}$. When $m_{A}$ differs from $m_{H}$ or when $\sin ^{2}(\alpha-\beta)$ is to some extent smaller than unity, the one-loop induced $\tau \rightarrow \mu \gamma$ process becomes important, and gives the most stringent bound on $\left|\kappa_{32}\right|^{2}$ of all the tau rare decay processes.

In Fig. 2-(a) and 2-(b), the similar contour plots are shown with assuming larger values of $m_{H}$ and $m_{H^{ \pm}}$; i.e., $m_{H}=m_{H^{ \pm}}=600 \mathrm{GeV}$ with $\sin (\alpha-\beta)=-0.9999$ for Fig. 2)(a) and $m_{H}=m_{A}=m_{H^{ \pm}}=600 \mathrm{GeV}$ for Fig. 2-(b), respectively. The magnitude of $\left|\kappa_{32}^{\max }\right|^{2}$ and its $\tan \beta$ dependence are similar to the case of Fig. 1 (a) and 1 1 (b), although the theoretical allowed regions become changed to the considerable extent.

In Figs. 1 and 2, the value of $\left|\kappa_{32}^{\max }\right|^{2}$ can be much larger than 100 in a wide range of the parameter region. One might think that such large values of $\left|\kappa_{3 i}\right|$ cannot be consistent with

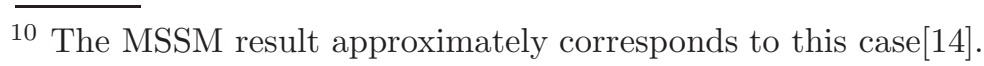


the unitarity argument for the LFV Yukawa couplings. However, it should be emphasized that the above figures show the contour plots for $\left|\kappa_{32}^{\max }\right|^{2}$ under the rare tau decay results, and not for $\left|\kappa_{32}\right|^{2}$. The region of $\left|\kappa_{32}^{\max }\right|^{2} \gtrsim 1$ should be taken as the area where $\left|\kappa_{32}\right|^{2}$ can be as large as $\mathcal{O}\left(10^{-2}-10^{-4}\right)$ easily. It is concluded that current results of the tau LFV decays do not give any substantial upper limit on $\left|\kappa_{32}\right|^{2}$ except for high $\tan \beta$ region $(\tan \beta \gtrsim 30)$.

Finally, we comment on the bound on $\left|\kappa_{31}\right|^{2}$, the LFV parameters for $\tau$-e mixing. Similar to $\tau$ - $\mu$ mixing, we can discuss $\left|\kappa_{31}^{\max }\right|^{2}$ comparing the data of $\tau \rightarrow e \eta, \tau \rightarrow e \mu^{+} \mu^{-}, \tau \rightarrow$ $e K^{+} K^{-}$and $\tau \rightarrow e \gamma$ listed in Table ! with the formulas given in Eqs. (14)-(20). These formulas for $\tau$-e mixing are common with $\tau$ - $\mu$ mixing except for the factor of $\left|\kappa_{3 i}\right|^{2}$, so that difference in contours of $\left|\kappa_{31}^{\max }\right|^{2}$ from those of $\left|\kappa_{32}^{\max }\right|^{2}$ only comes from that in the data. In Table I. the experimental limit for the branching ratio of $\tau \rightarrow$ en is about 1.5 times weaker than that of $\tau \rightarrow \mu \eta$, while that of $\tau \rightarrow e \gamma$ is 5.7 times relaxed as compared to that of $\tau \rightarrow \mu \gamma$. Moreover, the upper limit on $\operatorname{Br}\left(\tau^{-} \rightarrow e^{-} K^{+} K^{-}\right)$is 1.8 times stronger than that on $\operatorname{Br}\left(\tau^{-} \rightarrow \mu^{-} K^{+} K^{-}\right)$. We have numerically confirmed that there are some regions where $\tau^{-} \rightarrow e^{-} K^{+} K^{-}$can give the most stringent bound on $\left|\kappa_{31}\right|^{2}$. Therefore, $\left|\kappa_{31}^{\max }\right|^{2}$ is determined from one of $\tau \rightarrow e \eta, \tau \rightarrow e \gamma$ and $\tau^{-} \rightarrow e^{-} K^{+} K^{-}$depending on parameter regions.

\section{LEPTON FLAVOR VIOLATING HIGGS BOSON DECAYS}

As shown in the previous section, the LFV Yukawa couplings can be tested only in the large $\tan \beta$ region by searching for rare tau decays. In order to cover the region unconstrained by rare tau decay results, we here consider LFV via the decay of the neutral Higgs bosons;

i.e., $\phi^{0} \rightarrow \tau^{ \pm} \ell_{i}^{\mp}\left(\phi^{0}=h, H\right.$ and $\left.A\right)$. Branching ratios for these decays are calculated 15, 25, 26, 27] to be

$$
\begin{aligned}
\operatorname{Br}\left(h \rightarrow \tau^{-} \ell_{i}^{+}\right) & =\frac{1}{16 \pi} \frac{m_{\tau}^{2} \cos ^{2}(\alpha-\beta)}{v^{2} \cos ^{4} \beta}\left|\kappa_{3 i}\right|^{2} \frac{m_{h}\left(1-\frac{m_{\tau}^{2}}{m_{h}^{2}}\right)^{2}}{\Gamma(h \rightarrow \text { all })}, \\
\operatorname{Br}\left(H \rightarrow \tau^{-} \ell_{i}^{+}\right) & =\frac{1}{16 \pi} \frac{m_{\tau}^{2} \sin ^{2}(\alpha-\beta)}{v^{2} \cos ^{4} \beta}\left|\kappa_{3 i}\right|^{2} \frac{m_{H}\left(1-\frac{m_{\tau}^{2}}{m_{H}^{2}}\right)^{2}}{\Gamma(H \rightarrow \text { all })} \\
\operatorname{Br}\left(A \rightarrow \tau^{-} \ell_{i}^{+}\right) & =\frac{1}{16 \pi} \frac{m_{\tau}^{2}}{v^{2} \cos ^{4} \beta}\left|\kappa_{3 i}\right|^{2} \frac{m_{A}\left(1-\frac{m_{\tau}^{2}}{m_{A}^{2}}\right)^{2}}{\Gamma(A \rightarrow \text { all })}
\end{aligned}
$$




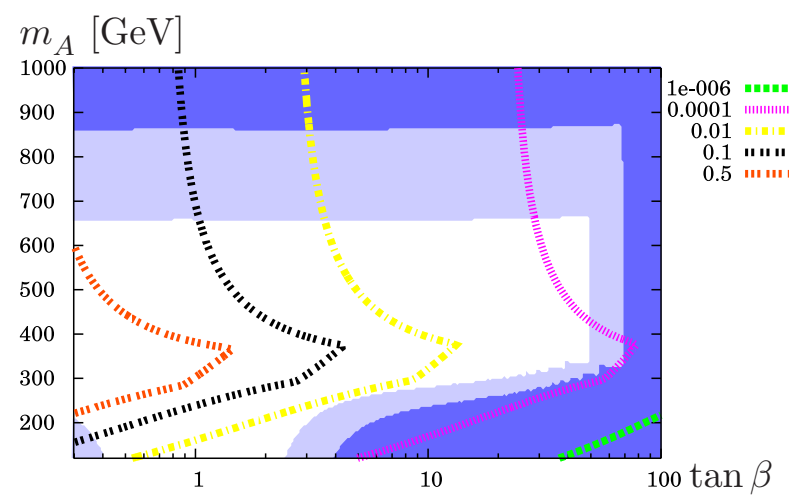

(a)

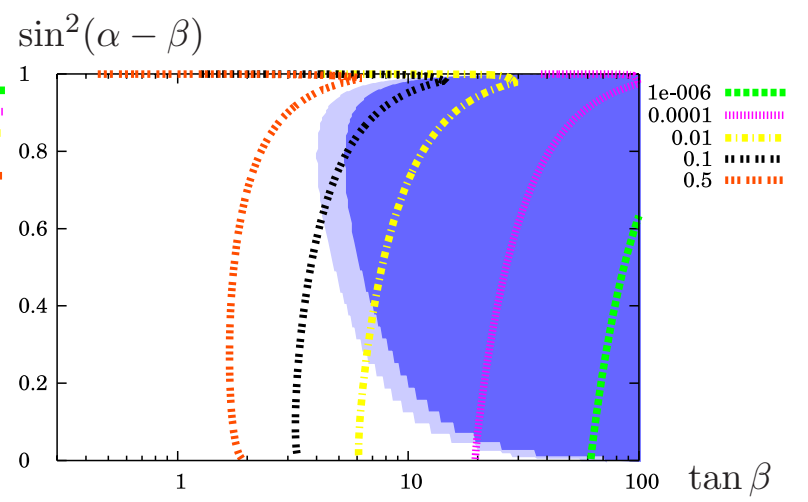

(b)

FIG. 3: Contour plots of $\operatorname{Br}\left(h \rightarrow \tau^{ \pm} \mu^{\mp}\right)_{\max }$, the possible maximal values for the branching ratio under the tau rare decay results, are shown (a) in the $\tan \beta-m_{A}$ plane and (b) in the $\tan \beta-\sin (\alpha-\beta)$ plane. The parameters are taken as the same as Figs. 1 1 (a) and 1 - b), respectively. The dark (light) shaded area indicates the excluded region by the theoretical requirements of Eqs. (8), (9) and (10) with $\xi=1(\xi=1 / 2)$.

where $\Gamma\left(\phi^{0} \rightarrow\right.$ all $)$ is the total width for corresponding neutral Higgs boson $\phi^{0}$. We here neglect terms of $\mathcal{O}\left(m_{\ell_{i}}^{2} / m_{\phi^{0}}^{2}\right)$. Branching ratios for $\phi^{0} \rightarrow \tau^{+} \ell_{i}^{-}$coincide with those for $\phi^{0} \rightarrow \tau^{-} \ell_{i}^{+}$given in Eqs. (24), (25) and (26). In the following, we concentrate on the decays into a $\tau$ - $\mu$ pair. We take the values of the SM parameters as $\alpha_{\text {em }}=0.007297$, $G_{F}=1.166 \times 10^{-5} \mathrm{GeV}^{-2}, m_{Z}=91.19 \mathrm{GeV}, m_{\tau}=1.777 \mathrm{GeV}, m_{\mu}=0.1057 \mathrm{GeV}, m_{b}=4.1$ $\mathrm{GeV}, m_{t}=174.3 \mathrm{GeV}$, and $m_{c}=1.15 \mathrm{GeV}$.

\section{A. The LFV decay of the lightest Higgs boson}

A search for the LFV decays $h \rightarrow \tau^{ \pm} \ell_{i}^{\mp}$ can give important information for extended Higgs sectors and thus for the structure of new physics, even when only $h$ is found and any other direct signals for the extended Higgs sector are not obtained by experiments. We here evaluate the possible maximal value of the branching ratio $\operatorname{Br}\left(h \rightarrow \tau^{-} \mu^{+}\right)_{\max }$ under the results of the rare tau decay search, by inserting $\left|\kappa_{32}^{\max }\right|^{2}$ of Eq. (23) into the $\left|\kappa_{32}\right|^{2}$ in Eq. (24). In calculation of $\Gamma(h \rightarrow$ all $)$, the decay modes of $b \bar{b}, c \bar{c}, \tau^{+} \tau^{-}, \gamma \gamma, g g, Z \gamma[\underline{40}]$ and $\tau^{ \pm} \ell_{i}^{\mp}$ are taken into account.

In Figs. 3.(a) and 3-(b), contours of $\operatorname{Br}\left(h \rightarrow \tau^{ \pm} \mu^{\mp}\right)_{\max }$, which is twice of $\operatorname{Br}(h \rightarrow$ $\left.\tau^{-} \mu^{+}\right)_{\max }$, are shown in the $\tan \beta-m_{A}$ plane and in the $\tan \beta-\sin ^{2}(\alpha-\beta)$ plane, respectively. 
The parameters are taken to be the same as those for Figs. 11 (a) and 1 $(b)$, respectively; i.e., (a) $m_{h}=120 \mathrm{GeV}, m_{H}=m_{H^{ \pm}}=350 \mathrm{GeV}$ and $\sin (\alpha-\beta)=-0.9999$, and (b) $m_{h}=120$ $\mathrm{GeV}$ and $m_{H}=m_{A}=m_{H^{ \pm}}=350 \mathrm{GeV}$, with $M$ to be scanned from 0 to $1000 \mathrm{GeV}$. We again show the excluded area from requirements of tree-level unitarity and vacuum stability as in the same way as Figs. 1 1 (a) and 1 1 (b). For low and moderate values of $\tan \beta$ $(\tan \beta \lesssim 30)$, where rare tau decay results cannot give substantial upper limit on $\left|\kappa_{32}\right|^{2}$, $\operatorname{Br}\left(h \rightarrow \tau^{ \pm} \mu^{\mp}\right)_{\max }$ can be sufficiently large. We find that the possible maximal values of the branching ratio can be greater than $\mathcal{O}\left(10^{-3}\right)$ in a wide rage of the allowed region under the theoretical conditions in Eqs. (8), (9) and (10).

In Fig. 44(a) and 4-(b), similar contour plots of $\operatorname{Br}\left(h \rightarrow \tau^{ \pm} \mu^{\mp}\right)$ max are shown for the same parameters as Figs. 22-(a) and 2-(b), respectively; i.e., (a) $m_{H}=m_{H^{ \pm}}=600 \mathrm{GeV}$ with $\sin (\alpha-\beta)=-0.9999$, and (b) $m_{H}=m_{A}=m_{H^{ \pm}}=600 \mathrm{GeV}$, with $M$ to be scanned from 0 to $1000 \mathrm{GeV}$. The excluded area from requirements of tree-level unitarity and vacuum stability are also shown. The possible maximal values for the branching ratio are similar to those in the case shown in Figs. 3.(a) and 3-(b). In the allowed region under the conditions in Eqs. (8), (91) and (10), $\operatorname{Br}\left(h \rightarrow \tau^{ \pm} \mu^{\mp}\right)_{\max }$ can be greater than $\mathcal{O}\left(10^{-2}\right)$.

For relatively lower $\tan \beta$ values, the experimental upper limits on $\left|\kappa_{32}\right|^{2}$ from rare tau decays are weaker, and $\operatorname{Br}\left(h \rightarrow \tau^{ \pm} \mu^{\mp}\right)$ can be sufficiently large $\left(\gtrsim \mathcal{O}\left(10^{-3}\right)\right.$ for $m_{h} \sim 120$ $\mathrm{GeV}$ ). It is expected that a sufficient number of such light $h$ can be produced at future colliders such as CERN LHC, currently planned International Linear Collider (ILC) and CERN CLIC. It has been pointed out that the decay process $h \rightarrow \tau^{ \pm} \mu^{\mp}$ can easily be detected at ILC with the luminosity of $1 \mathrm{ab}^{-1}$, when $m_{h} \sim 120 \mathrm{GeV}$ and $\operatorname{Br}\left(h \rightarrow \tau^{ \pm} \mu^{\mp}\right) \gtrsim$ $\mathcal{O}\left(10^{-3}\right)^{11}$ via the Higgsstrahlung process by using the recoil momentum of $Z$ boson [27]. Therefore, the LFV search via the decay $h \rightarrow \tau^{ \pm} \mu^{\mp}$ at ILC can be complementary to that via rare tau decays at (super) B factories, and the both cover a wide region of the parameter space of the lepton flavor violating THDM.

${ }^{11}$ Below that value, the signal would be seriously suffered from the backgrounds. In particular, events from $h \rightarrow \mu^{+} \mu^{-}$and $h \rightarrow \tau^{+} \tau^{-}$would be difficult to be separated from the signal. The branching ratio of $h \rightarrow \mu^{+} \mu^{-}$is approximately proportional to $m_{\mu}^{2} /\left(N_{c} m_{b}^{2}\right)$ for a relatively light $h$, with $N_{c}$ being the color factor; i.e., $\operatorname{Br}\left(h \rightarrow \mu^{+} \mu^{-}\right) \sim 3 \times 10^{-4}$. For a large $\tan \beta$ value with $\sin ^{2}(\alpha-\beta) \ll 1$, the maximal value of $\operatorname{Br}\left(h \rightarrow \tau^{ \pm} \mu^{\mp}\right)$ becomes smaller and can be comparable to $\operatorname{Br}\left(h \rightarrow \mu^{+} \mu^{-}\right)$. A simulation study is necessary to clarify this point. 


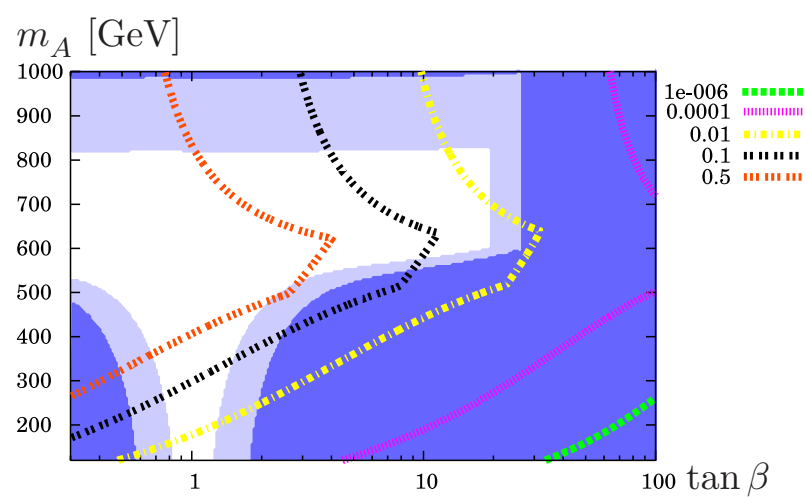

(a)

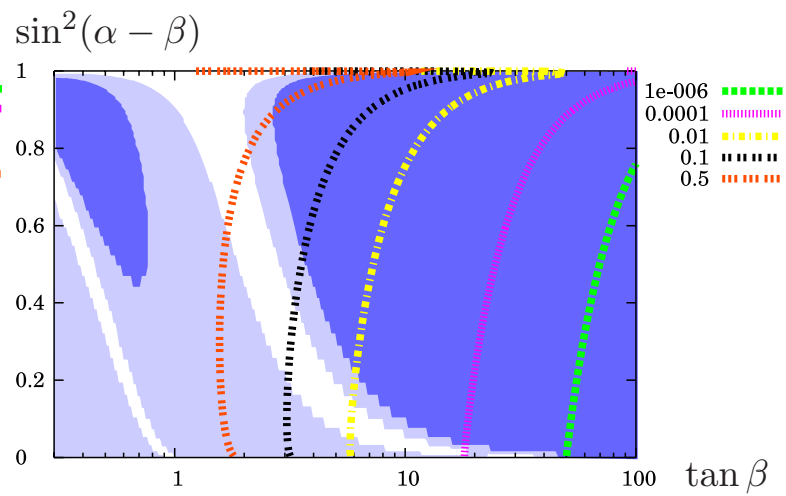

(b)

FIG. 4: Contour plots of $\operatorname{Br}\left(h \rightarrow \tau^{ \pm} \mu^{\mp}\right)_{\max }$ similar to Figs. 3-(a) and 3-(b) are shown (a) in the $\tan \beta-m_{A}$ plane and (b) in the $\tan \beta-\sin ^{2}(\alpha-\beta)$ plane with the parameters taken as the same as Figs. 2-(a) and 2-(b), respectively. The dark (light) shaded area indicates the excluded region by the theoretical requirements of Eqs. (8), (9) and (10) with $\xi=1(\xi=1 / 2)$.

\section{B. The LFV decay of Heavier Higgs bosons}

Next we discuss branching ratios for the LFV decays of heavier Higgs bosons, $H / A \rightarrow$ $\tau^{ \pm} \ell_{i}^{\mp}$, using Eqs. (25) and (26) under the current data of LFV rare tau decays. In the THDM, there are many possible decay modes for $H$ depending on the mass spectrum; i.e., $h h, A A, h A, h \gamma, h Z, A \gamma, A Z, H^{+} H^{-}$and $H^{ \pm} W^{\mp}$ as well as $f \bar{f}(f=t, b, c, \tau), W^{ \pm} W^{\mp}$, $Z Z, Z \gamma, \gamma \gamma$ and $g g$. The last three modes as well as $h A, h \gamma, A \gamma$ and $h Z$ appear through the one-loop induced couplings ${ }^{12}$. Those for $A$ are $h Z, H Z, H^{+} H^{-}, H^{ \pm} W^{\mp}$ and $f \bar{f}$ at the tree level as well as $h h, h H, H H, h \gamma, H \gamma, W^{ \pm} W^{\mp}, Z Z, Z \gamma, \gamma \gamma$ and $g g$ at the one loop level [40].

The branching ratios for $H / A \rightarrow \tau^{ \pm} \ell_{i}^{\mp}$ are sensitive to the masses of all the Higgs bosons. Here we consider the case of $\sin (\alpha-\beta)=-1$ and $m_{H}=m_{A}=m_{H^{ \pm}}\left(\equiv m_{\Phi}\right)$. As discussed in Sec. II, the $\rho$ parameter constraint is satisfied for this choice. From the $b \rightarrow s \gamma$ results, $m_{\Phi}$ is taken to be greater than $350 \mathrm{GeV}$. As also discussed in Sec. II, $M$ determines the decoupling property of heavier Higgs bosons. Although the branching ratios $\operatorname{Br}\left(H / A \rightarrow \tau^{ \pm} \ell_{i}^{\mp}\right)$ are insensitive to $M$ in the present parameter set, its value strongly affects the allowed parameter

\footnotetext{
${ }^{12}$ In the numerical analysis, we included contributions from these $Z \gamma, \gamma \gamma$ and $g g$ modes in addition to all the tree level modes, but neglected the other loop-induced modes.
} 


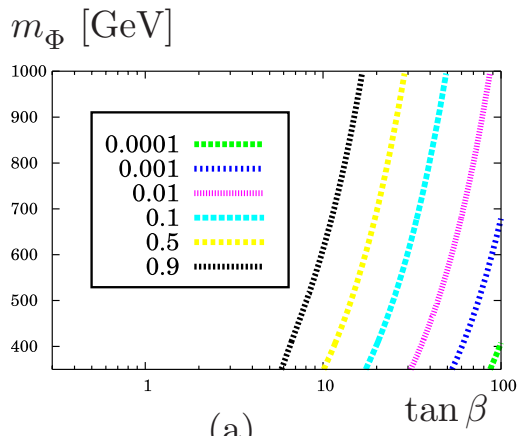

(a)

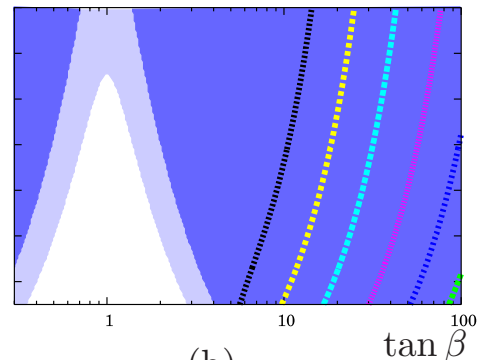

(b)

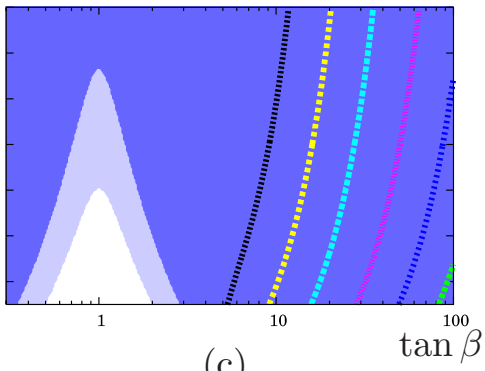

(c)

FIG. 5: Contour plots of $\operatorname{Br}\left(H \rightarrow \tau^{ \pm} \mu^{\mp}\right)_{\max }$ are shown in the $\tan \beta-m_{\Phi}$ plane $\left(m_{\Phi} \equiv m_{H}=\right.$ $\left.m_{A}=m_{H^{ \pm}}\right)$for $m_{h}=120 \mathrm{GeV}$ and $\sin (\alpha-\beta)=-1$ with (a) $M=m_{\Phi}$, (b) $M=m_{\Phi} / \sqrt{2}$, and (c) $M=0$. The dark (light) shaded area indicates the excluded region by the theoretical requirements of Eqs. (8), (9) and (10) with $\xi=1(\xi=1 / 2)$.

region under the theoretical conditions of Eqs. (8) and (9). Notice that couplings of $H$ are similar to those of $A$ for $\sin (\alpha-\beta)=-1$ where there are no $H V V$ couplings. Hence we show the results only for the LFV decays of $H$ below. In a general case, the branching ratio of $H \rightarrow \tau^{ \pm} \mu^{\mp}$ tends to be smaller than that of $A \rightarrow \tau^{ \pm} \mu^{\mp}$ due to the contribution from the modes $H \rightarrow V V$.

In Figs. 5-(a), 5-(b) and 5-(c), contour plots of $\operatorname{Br}\left(H \rightarrow \tau^{ \pm} \mu^{\mp}\right)_{\max }$, the upper limit of $\operatorname{Br}\left(H \rightarrow \tau^{ \pm} \mu^{\mp}\right)$ under the rare tau decay results, are shown in the $\tan \beta-m_{\Phi}$ plane for $M=m_{\Phi}, m_{\Phi} / \sqrt{2}$ and 0 , respectively. As expected, the contours are insensitive to the values of $M$, and approximately the same in Figs. 5.(a), 5.(b) and 5.(c). It is shown that $\operatorname{Br}\left(H \rightarrow \tau^{ \pm} \mu^{\mp}\right)_{\max }$ can be larger than $10^{-3}$ except for large $\tan \beta$ values with relatively small $m_{\Phi}$. Therefore, it turns out to be no substantial upper limit on the $\operatorname{Br}\left(H \rightarrow \tau^{ \pm} \mu^{\mp}\right)$ in the relatively low $\tan \beta$ region $(\tan \beta \lesssim 20)$ from the LFV rare tau decay results. When $M$ is smaller than $m_{\Phi}$, where the heavier Higgs boson partially receive their masses from the vacuum expectation value, the allowed parameter region is strongly constrained by the requirements of vacuum stability and perturbative unitarity. In particular, for $M=0$ (Fig. 5. (c)), the allowed region is limited only the area of around $\tan \beta \sim 1$ and $m_{\Phi} \lesssim 600$ $\mathrm{GeV}$. 
The extra Higgs bosons $\left(H, A\right.$ and $\left.H^{ \pm}\right)$are expected to be searched at the LHC. The signal of $g g \rightarrow H / A \rightarrow \tau^{ \pm} \mu^{\mp}$ may be detectable at LHC with high luminosity $\left(100 \mathrm{fb}^{-1}\right)$

when $\operatorname{Br}\left(H / A \rightarrow \tau^{ \pm} \mu^{\mp}\right)$ is greater than $10^{-2}$ for $m_{H / A} \sim 350 \mathrm{GeV}$ and $\tan \beta=45[26]$. However the rate is rapidly reduced for smaller values of $\tan \beta$ and for larger values of $m_{H / A}$. Further feasibility study is necessary.

\section{CONCLUSIONS}

Lepton flavor violating decays of Higgs bosons have been studied in the framework of the THDM, in which LFV couplings are introduced as a deviation from Model II Yukawa interaction in the lepton sector. The model parameters are constrained by requirements of tree-level unitarity and vacuum stability, and also from the experimental results. The parameters $\left|\kappa_{3 i}\right|^{2}$ in LFV Yukawa interactions are bounded from above by using the current data for rare tau LFV decays. Each process is mediated by the different combination of the Higgs bosons, so that the data for each of them provides independent information to the lepton flavor violating THDM. It has been found that among the rare tau decay data those for $\tau \rightarrow \ell_{i} \eta$ and $\tau \rightarrow \ell_{i} \gamma$ give the most stringent upper limits on $\left|\kappa_{3 i}\right|^{2}$ in a wide range of the parameter space.

In the large $\tan \beta$ region ( $\tan \beta \gtrsim 30$ ), the upper limit on $\left|\kappa_{3 i}\right|^{2}$ due to the rare tau decay data turns out to be substantial and comparable with the value predicted by assuming some fundamental theories such as SUSY. The upper limit would be improved in future by about one order of magnitude at the experiment at (super) B factories. For smaller values of $\tan \beta$, the upper limit is rapidly relaxed, and no more substantial constraint is obtained from the rare tau decay results.

We have shown that a search for the LFV decays $\phi^{0} \rightarrow \tau^{ \pm} \ell_{i}^{\mp}$ of neutral Higgs bosons $\left(\phi^{0}=h, H\right.$ and $\left.A\right)$ can be useful to further constrain the LFV Yukawa couplings at future collider experiments. In particular, the LFV decays of the lightest Higgs boson can be one of the important probes to find the evidence for the extended Higgs sector when the SM-like situation would be preferred by the data at forthcoming collider experiments. The branching ratio for $h \rightarrow \tau^{ \pm} \mu^{\mp}$ can be larger than $\mathcal{O}\left(10^{-3}\right)$ except for the high $\tan \beta$ region under the constraints from the current experimental data and also from the theoretical requirements. At ILC (and in case at LHC), such a size of the branching fractions can be 
tested. Therefore, we conclude that the search of LFV in the Higgs boson decay at future colliders can further constrain the LFV Yukawa couplings especially in the relatively small $\tan \beta$ region $(\tan \beta \lesssim 30)$, where rare tau decay data cannot reach.

\section{Acknowledgments}

The authors would like to thank Yasuhiro Okada for speculative comments, Eric Torrence, Paride Paradisi for helpful comments, and Eiichi Takasugi, Koichi Matsuda, Tetsuo Shindou for valuable discussions. S.K was supported, in part, by Grants-in-Aid of the Ministry of Education, Culture, Sports, Science and Technology, Government of Japan, Grant No. 17043008. T.O. was supported, in part, by JSPS Research Fellowship for Young Scientists, No. 15-03693.

[1] Y. Kuno and Y. Okada, Rev. Mod. Phys. 73, 151 (2001).

[2] Y. Enari et al., the Belle Collaboration, arXiv:hep-ex/0503041.

[3] Y. Yusa, Nucl. Phys. B (Proc. Suppl.) 144, 173 (2005).

[4] M. Hodkinson (on behalf of the BaBar Collaboration), Nucl. Phys. B (Proc. Suppl.) 144, 167 (2005).

[5] Y. Yusa et al., the Belle Collaboration, Phys. Lett. B 589, 103 (2004).

[6] B. Aubert et al., the BaBar Collaboration, Phys. Rev. Lett. 92, 121801 (2004).

[7] K. Hayasaka et al., the Belle Collaboration, Phys. Lett. B 613, 20 (2005).

[8] K. Abe et al., the Belle Collaboration, Phys. Rev. Lett. 92, 171802 (2004).

[9] B. Aubert et al., the BaBar Collaboration, arXiv:hep-ex/0502032,

[10] R. Kitano, M. Koike, S. Komine, and Y. Okada, Phys. Lett. B 575, 300 (2003).

[11] R.A. Diaz, R. Martinez, and J.-A. Rodriguez, Phys. Rev. D 63, 096007 (2001).

[12] K.S. Babu and C. Kolda, Phys. Rev. Lett. 89, 241802 (2002).

[13] A. Dedes, J. Ellis, and M. Raidal, Phys. Lett. B 549, 159 (2002).

[14] M. Sher, Phys. Rev. D 66, 057301 (2002).

[15] A. Brignole and A. Rossi, Phys. Lett. B 566, 217 (2003); Nucl. Phys. B 701, 3 (2004).

[16] T.P. Cheng and M. Sher, Phys. Rev. D 35, 3484 (1987).

[17] J.L. Diaz-Cruz and J.J. Toscano, Phys. Rev. D 62, 116005 (2002); J.L. Diaz-Cruz, R. Noriega- 
Papaqui, and A. Rosado, Phys. Rev. D 71, 015014 (2005).

[18] E.O. Iltan, JHEP 0402, 065 (2004); JHEP 0408, 020 (2004); hep-ph/0504013.

[19] D. Black, T. Han, H.-J. He, and M. Sher, Phys. Rev. D 66, 053002 (2002).

[20] D. Guetta, J.M. Mira, and E. Nardi, Phys. Rev. D 59, 034019 (1999).

[21] A.G. Akeroyd et al., Super KEKB Letter of Intent, KEK Report 04-4, arXiv:hep-ex/0406071.

[22] P. Paradisi, arXiv:hep-ph/0508054.

[23] J. Ellis, J. Hisano, M. Raidal, and Y. Shimizu Phys. Rev. D 66, 115013 (2002).

[24] A. Pilaftsis, Phys. Lett. B 285, 68 (1992).

[25] E. Arganda, A.M. Curiel, M.J. Herrero, and D. Temes, Phys. Rev. D 71, 035011 (2005).

[26] K.A. Assamagan, A. Deandrea, and P.-A. Delsart, Phys. Rev. D 67, 035001 (2003).

[27] S. Kanemura, K. Matsuda, T. Ota, T. Shindou, E. Takasugi, and K. Tsumura, Phys. Lett. B 599, 83 (2004).

[28] M. Sher, Phys. Lett. B 487, 151 (2000); U. Cotti, M. Pineda, and G. Tavares-Velasco, arXiv:hep-ph/0501162,

[29] M. Sher and I. Turan, Phys. Rev. D 69, 017302 (2004).

[30] S. Kanemura, Y. Kuno, M. Kuze, and T. Ota, Phys. Lett. B 607, 165 (2005); Nucl. Phys. B (Proc. Suppl.) 144, 268 (2005).

[31] N.G. Deshpande and E. Ma, Phys. Rev. D 18, 2574 (1978); S. Kanemura, T. Kasai, and Y. Okada, Phys. Lett. B 471, 182 (1999); S. Nie and M. Sher, Phys. Lett. B 449, 89 (1999).

[32] B.W. Lee, C. Quigg, and H.B. Thacker, Phys. Rev. Lett. 38, 883 (1977); Phys. Rev. D 16, 1519 (1977).

[33] S. Kanemura, T. Kubota, and E. Takasugi, Phys. Lett. B 313, 155 (1993).

[34] H. Hüffel and G. Pocsik, Z. Phys. C 8, 13 (1981); J. Maalampi, J. Sirkka, and I. Vilja, Phys. Lett. B 265, 371 (1991); A. Akeroyd, A. Arhrib, and E.-M. Naimi, Phys. Lett. B 490, 119 (2000); I.F. Ginzburg and I.P. Ivanov, arXiv:hep-ph/0312374.

[35] LEP Electroweak Working Group, http://lepewwg.web.cern.ch/LEPEWWG/.

[36] P.H. Chankowski, M. Krawczyk, and J. Zochowski, Euro. Phys. J. C 11, 661 (1999); H.E. Haber and A. Pomarol, Phys. Lett. B 302, 435 (1993); A. Pomarol and R. Vega, Nucl. Phys. B 413, 3 (1994).

[37] P. Koppenburg et al., the Belle Collaboration, Phys. Rev. Lett. 93, 061803 (2004).

[38] M. Ciuchini, G. Degrassi, P. Gambini, and G.F. Giudice, Nucl. Phys. B 527, 21 (1998); 
P. Ciafaloni, A. Romanino, and A. Strumia, Nucl. Phys. B 524, 361 (1998); F. Borzumati and G. Greub, Phys. Rev. D 58, 074004 (1998); T.M. Aliev and E.O. Iltan, Phys. Rev. D 58, 095014 (1998).

[39] S. Glashow and S. Weinberg, Phys. Rev. D 15, 1958 (1977).

[40] J.F. Gunion, H.E. Haber, G. Kane, and S. Dawson, The Higgs Hunter's Guide, Perseus Publishing, Cambridge, MA, 1990.

[41] W.S. Hou, Phys. Lett. B 296, 179 (1992); D. Atwood, L. Reina, and A. Soni, Phys. Rev. D 53, 1199 (1996); Phys. Rev. D 55, 3156 (1997); W.S. Hou, G.L. Lin, and C.Y. Ma, Phys. Rev. D 56, 7434 (1997); S. Béjar, J. Guasch, and J. Solá, Nucl. Phys. B 675, 270 (2003); A. Cordero-Cid, M.A. Rérez, G. Tavares-Velasco, and J.J. Toscano, Phys. Rev. D 70, 074003 (2004); A. Arhrib, Phys. Lett. B 612, 263 (2005).

[42] A.M. Curiel, M.J. Herrero, and D. Temes, Phys. Rev. D 67, 075008 (2003); A.M. Curiel, M.J. Herrero, W. Hollik, F. Merz, and S. Peñaranda, Phys. Rev. D 69, 075009 (2004); S. Béjar, F. Dilé, J. Guasch, and J. Solá, JHEP 0408, 018 (2004).

[43] F. Borzumati and A. Masiero, Phys. Rev. Lett. 57, 961 (1986); J. Hisano, T. Moroi, K. Tobe, M. Yamaguchi, and T. Yanagida, Phys. Lett. B 357, 579 (1995); J. Hisano, T. Moroi, K. Tobe, and M. Yamaguchi, Phys. Rev. D 53, 2442 (1996).

[44] A. Zee, Phys. Lett. B 93, 389 (1980); Erratum ibid 95, 461 (1980); S. Kanemura, T. Kasai, G.-L. Lin, Y. Okada, J.-J. Tseng, and C.-P. Yuan, Phys. Rev. D 64, 053007 (2001); K. Cheung and O. Seto, Phys. Rev. D 69, 113009 (2004).

[45] S. Kanemura, S. Kiyoura, Y. Okada, E. Senaha, and C.-P. Yuan, Phys. Lett. B 558, 157 (2003); S. Kanemura, Y. Okada, E. Senaha, and C.-P. Yuan, Phys. Rev. D 70, 115002 (2004).

[46] J.F. Gunion and H.E. Haber, Phys. Rev. D 67, 075019 (2003).

[47] I.F. Ginzburg, M. Krawczyk, and P. Osland, arXiv:hep-ph/0101208, Nucl.Instrum.Meth. A472 149 (2001).

[48] V. Barger, J.L. Hewett, and R.J.N. Phillips, Phys. Rev. D 41, 3421 (1990). 